\title{
Invariant Contrast Parameters of PolInSAR Homogenous RVoG Model
}

\author{
Philippe Réfrégier, Antoine Roueff, Aurélien Arnaubec, and Pascale C. Dubois-Fernandez, Senior Member, IEEE
}

\begin{abstract}
It has been shown that the Cramer-Rao bound (CRB) can be helpful to characterize vegetation and ground height estimations based on the homogenous random volume over ground (RVoG) model and polarimetric interferometric SAR techniques. However, this model is a function of 20 unknown parameters, which makes the performance analysis a tedious task. We show that the group invariance property of the RVoG model can greatly reduce the complexity of the analysis since the CRB of the vegetation and ground heights only depends on four unknown parameters instead of 20 . Furthermore, for the considered situations analyzed in this letter, only three of these four parameters have a nonnegligible influence and can be interpreted as contrast parameters.
\end{abstract}

Index Terms-Cramer-Rao bound (CRB), polarimetric interferometric SAR (PolInSAR), random volume over ground (RVoG) model.

\section{INTRODUCTION AND BACKGROUND}

$\mathbf{P}$ OLARIMETRIC interferometric SAR (PolInSAR) techniques consist in illuminating a scene with electromagnetic waves with two different polarization states and receiving the backscattered wave at two close locations [1], [2]. Let $S_{j}^{X Y}$ denote the measured amplitude along the polarization $Y$ of the receiver number $j$ when the scene is illuminated with a transmit polarization $X$. Assuming that the reciprocity principle holds, the component $S_{j}^{V H}$ is equal to $S_{j}^{H V}$ and is thus not mentioned [1], [2]. With two orthogonal transmit polarization states $(H, V)$, it is possible to obtain the vector response $\boldsymbol{u}_{j}^{T}=$ $\left(S_{j}^{H H}, \sqrt{2} S_{j}^{H V}, S_{j}^{V V}\right)$, where ${ }^{T}$ denotes the transpose. With two polarimetric receivers, the response can be written with the complex vector $\boldsymbol{k}=\left(\boldsymbol{u}_{1}^{T}, \boldsymbol{u}_{2}^{T}\right)^{T}$. This vector $\boldsymbol{k}$ is assumed to

P. Réfrégier and A. Roueff are with Centrale Marseille, CNRS, Aix Marseille Université, Institut Fresnel, Unité Mixte de Recherche 7249, 13013 Marseille, France.

A. Arnaubec was with the Imager Radar and Testing Unit, Department of Electromagnetism and Radar, ONERA, 13661 Salon-de-Provence, France, and also with Centrale Marseille, CNRS, Aix Marseille Université, Institut Fresnel, Unité Mixte de Recherche 7249, 13013 Marseille, France. He is now with the Underwater System Department, IFREMER, 83507 La Seyne-sur-Mer, France.

P. C. Dubois-Fernandez is with the Department of Electromagnetism and Radar, ONERA, 13661 Salon-de-Provence, France. be distributed with a circular zero-mean Gaussian distribution characterized by its covariance matrix $\Upsilon$, i.e.,

$$
\Upsilon=\left(\begin{array}{cc}
\boldsymbol{T}_{1} & \boldsymbol{\Omega} \\
\boldsymbol{\Omega}^{\dagger} & \boldsymbol{T}_{2}
\end{array}\right)
$$

where $\dagger$ corresponds to the transpose complex conjugate, where $\boldsymbol{T}_{j}=\left\langle\boldsymbol{u}_{j} \boldsymbol{u}_{j}^{\dagger}\right\rangle$ for $j=1,2, \boldsymbol{\Omega}=\left\langle\boldsymbol{u}_{1} \boldsymbol{u}_{2}^{\dagger}\right\rangle$, and where \langle\rangle is the statistical average.

The random volume over ground (RVoG) model [3] has been proposed to analyze the vegetation response. The interaction of the incident wave with the vegetation volume (respectively, the ground) is thus described with the covariance matrix $\boldsymbol{T}_{\mathrm{vol}}$ (respectively, $\boldsymbol{T}_{\text {gro }}$ ) and the attenuation $\sigma_{v}$. The PolInSAR system geometry is characterized by the height sensitivity $k_{z}$ and the incident angle $\beta$. The RVoG model can thus be written as [3]-[6]

$$
\begin{aligned}
& \boldsymbol{T}=\boldsymbol{T}_{1}=\boldsymbol{T}_{2}=I_{1} \boldsymbol{T}_{\mathrm{vol}}+a \boldsymbol{T}_{\text {gro }} \\
& \boldsymbol{\Omega}=e^{i k_{z} z_{g}}\left(I_{2} \boldsymbol{T}_{\mathrm{vol}}+a \boldsymbol{T}_{\text {gro }}\right)
\end{aligned}
$$

with $I_{1}=\left(1-e^{-\alpha h_{v}}\right) / \alpha, I_{2}=\left(e^{i k_{z} h_{v}}-e^{-\alpha h_{v}}\right) /\left(i k_{z}+\alpha\right)$, $a=e^{-\alpha h_{v}}$, and $\alpha=2 \sigma_{v} / \cos \beta$. In this letter, we consider single baseline P-band PolInSAR systems, and we assume as in [7] that the attenuation $\sigma_{v}$ is polarization independent and a priori known. The nine parameters of $\boldsymbol{T}_{\mathrm{vol}}$, noted $t_{\mathrm{vol}, 1}, \ldots, t_{\mathrm{vol}, 9}$, and the nine parameters of $\boldsymbol{T}_{\mathrm{gro}}$, noted $t_{\mathrm{gro}, 1}, \ldots, t_{\mathrm{gro}, 9}$, are assumed unknown. With these assumptions, the vegetation height $h_{v}$ and the ground height $z_{g}$ can be estimated [7], [8], with the estimation of $\mathbf{\Upsilon}$ in homogenous regions, so that the elements of the set $\mathcal{K}=\left\{\boldsymbol{k}_{1}, \boldsymbol{k}_{2}, \ldots, \boldsymbol{k}_{N}\right\}$ of $N$ measured vectors $\boldsymbol{k}_{j}$ can be considered distributed with the same probability density. Although the random volume model leads to a constrained parametric form of $\boldsymbol{T}_{\mathrm{vol}}$ [2], no constraint are assumed in this letter on this matrix because such a constraint is usually not taken into account for the estimation of $h_{v}$ and $z_{g}[5]-[7]$.

The Cramer-Rao bounds $\mathrm{CRB}\left[h_{v}\right]$ and $\mathrm{CRB}\left[z_{g}\right]$ [8], [9] correspond to a lower bound for the variance of the estimation of $h_{v}$ and $z_{g}$, respectively, with unbiased estimators (i.e., such that the mean value of the estimated quantity is equal to the true value) [10]. These CRBs are function of the 20 scalar unknown parameters $\boldsymbol{\Theta}^{T}=\left(\theta_{1}, \theta_{2}, \ldots, \theta_{20}\right)$, where $\boldsymbol{\Theta}^{T}=\left(h_{v}, z_{g}, t_{\mathrm{vol}, 1}, \ldots, t_{\mathrm{vol}, 9}, t_{\mathrm{gro}, 1}, \ldots, t_{\mathrm{gro}, 9}\right)$. This dependence of the CRB on a large number of parameters is a source of complexity. Indeed, for a fixed PolInSAR configuration (specified by $k_{z}$ and $\beta$ ) and for a known value of $\sigma_{v}$, a 
full analysis of the precision on $h_{v}$ and $z_{g}$ would require to examine $\mathrm{CRB}\left[h_{v}\right]$ and $\mathrm{CRB}\left[z_{g}\right]$ in a mathematical space of dimension 20. However, the analysis of the estimations of $h_{v}$ and $z_{g}$ based on the representation of the region of coherence [1], [2], [5]-[7] corresponds to a parametric model with less than 20 parameters, and the demonstration that this approach can be efficient [8], [9] suggests that the rigorous dimension of the problem may be indeed lower than 20. As shown in the next section, group invariance properties can be helpful to rigorously show that $\mathrm{CRB}\left[h_{v}\right]$ and $\mathrm{CRB}\left[z_{g}\right]$ only depend on four unknown parameters, which greatly simplify the analysis.

\section{INVARIANT PARAMETERS}

Statistical invariance properties have been widely used in statistics [11], [12] and more recently for the analysis of polarimetric image processing performance [13]-[15]. Let us consider that the $N$ measurements of the set $\mathcal{K}$ have been transformed into $\boldsymbol{k}_{j}^{\prime}=\boldsymbol{M} \boldsymbol{k}_{j} \forall j \in\{1, \ldots, N\}$, where

$$
\boldsymbol{M}=\left[\begin{array}{cc}
\boldsymbol{B} & \mathbf{0} \\
\mathbf{0} & e^{i k_{z} z} \boldsymbol{B}
\end{array}\right]
$$

where $z$ is a real scalar, $\mathbf{0}$ is the $3 \times 3$ zero matrix, and $\boldsymbol{B}$ is a nonsingular $3 \times 3$ matrix. It is easy to see that the new set of measurements $\mathcal{K}^{\prime}=\left\{\boldsymbol{k}_{1}^{\prime}, \ldots, \boldsymbol{k}_{N}^{\prime}\right\}$ could have been also obtained with an RVoG model of parameters $h_{v}^{\prime}=h_{v}$, $z_{g}^{\prime}=z_{g}-z, \boldsymbol{T}_{\mathrm{vol}}^{\prime}=\boldsymbol{B} \boldsymbol{T}_{\mathrm{vol}} \boldsymbol{B}^{\dagger}$, and $\boldsymbol{T}_{\mathrm{gro}}^{\prime}=\boldsymbol{B} \boldsymbol{T}_{\mathrm{gro}} \boldsymbol{B}^{\dagger}$. Thus, the application of (3) transforms an RVoG problem into another one. This property thus shows that transformations described by (3) leave invariant the family of RVoG models [11], [12]. It can be seen (see the Appendix) that the CRBs for the estimation of $h_{v}$ and $z_{g}$ are equal for the RVoG model with parameters $h_{v}, z_{g}, \boldsymbol{T}_{\mathrm{vol}}$, and $\boldsymbol{T}_{\text {gro }}$ and with parameters $h_{v}, z_{g}-z, \boldsymbol{T}_{\text {vol }}^{\prime}$, and $\boldsymbol{T}_{\text {gro }}^{\prime}$. Since $z$ is deterministic and can have any values (in the limit of nonambiguous values), the CRB is expected not to depend on $z_{g}$. Furthermore, assuming that $\boldsymbol{T}_{\mathrm{vol}}$ is nonsingular, the choice $\boldsymbol{B}=\boldsymbol{U} \boldsymbol{T}_{\mathrm{vol}}^{-1 / 2}$, where $\boldsymbol{U}$ is the unitary matrix that diagonalizes the Hermitian matrix $\boldsymbol{T}_{\mathrm{vol}}^{-1 / 2} \boldsymbol{T}_{\mathrm{gro}} \boldsymbol{T}_{\mathrm{vol}}^{-1 / 2}$, leads to $\boldsymbol{T}_{\text {vol }}^{\prime}=\boldsymbol{I}_{d}$, where $\boldsymbol{I}_{d}$ is the $3 \times 3$ identity matrix and $\boldsymbol{T}_{\text {gro }}^{\prime}=$ $\operatorname{Diag}\left[\lambda_{1}, \lambda_{2}, \lambda_{3}\right]$, where $\operatorname{Diag}\left[\lambda_{1}, \lambda_{2}, \lambda_{3}\right]$ is the diagonal matrix with positive diagonal values $\lambda_{1}, \lambda_{2}, \lambda_{3}$ that are the eigenvalues of $\boldsymbol{T}_{\mathrm{vol}}^{-1 / 2} \boldsymbol{T}_{\text {gro }} \boldsymbol{T}_{\text {vol }}^{-1 / 2}$.

The obtained RVoG model, which corresponds to parameters $h_{v}^{\prime}=h_{v}, z_{g}^{\prime}=0, \boldsymbol{T}_{\mathrm{vol}}^{\prime}=\boldsymbol{I}_{d}$, and $\boldsymbol{T}_{\text {gro }}^{\prime}=\operatorname{Diag}\left[\lambda_{1}, \lambda_{2}, \lambda_{3}\right]$, has only four independent parameters, and it is thus expected that $\mathrm{CRB}\left[h_{v}\right]$ and $\mathrm{CRB}\left[z_{g}\right]$ depend only on these four parameters. Thus, it is sufficient to explore a mathematical space of dimension 4 instead of 20 in order to analyze the performance of the estimation of $h_{v}$ and $z_{g}$ with a homogenous RVoG model and PolInSAR systems. An illustration of the invariance of $\operatorname{CRB}\left(h_{v}\right)$ and $\operatorname{CRB}\left(z_{g}\right)$ for different $z_{g}$ values can be obtained with the region of coherence [6] since changing $z_{g}$ corresponds to a rotation of this region without modifying its shape.

The property of invariance is illustrated in Fig. 1(a), where the CRB values are shown when the sample size is $N=100$.

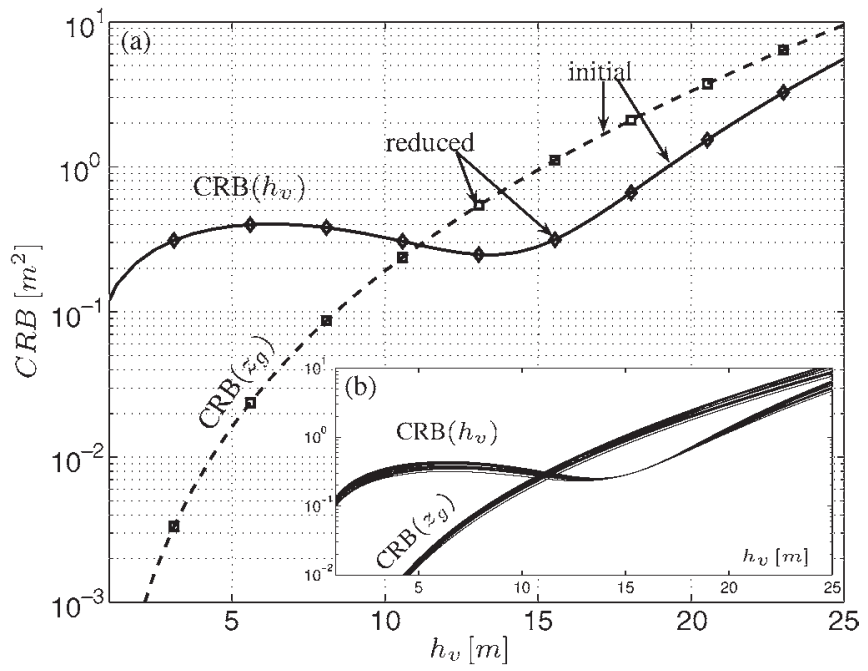

Fig. 1. (a) Continuous: CRB for $h_{v}$ with the initial model. Diamonds: CRB for $h_{v}$ with the reduced model. Dashed: CRB for $z_{g}$ with the initial model. Squares: CRB for $z_{g}$ with the reduced model. (b) Inset: CRB as a function of $h_{v}$ for different $x \in[0,1]$ when $\lambda_{2}(x)=x \lambda_{1}(x)+(1-x) \lambda_{3}(x), \mathcal{A}=0.59$, and $\mathcal{E}=71.2$.

Matrices $\boldsymbol{T}_{\text {gro }}$ and $\boldsymbol{T}_{\mathrm{vol}}$ (the physical unit of $\boldsymbol{T}_{\mathrm{vol}}$ is $\mathrm{m}^{-1}$; see [8]) were equal to

$$
\begin{aligned}
\boldsymbol{T}_{\text {gro }} & =\left(\begin{array}{ccc}
5.43 & 2.03+i 1.06 & 1.06+i 0.318 \\
2.03-i 1.06 & 4.94 & 0.0886+i 0.452 \\
1.06-i 0.318 & 0.0886-i 0.452 & 2.17
\end{array}\right) \\
\boldsymbol{T}_{\mathrm{vol}} & =0.01\left(\begin{array}{ccc}
23.9 & -3+i 0.793 & 3.59+i 1.27 \\
-3-i 0.793 & 16.8 & -0.582+i 2.2 \\
3.59-i 1.27 & -0.582-i 2.2 & 13.7
\end{array}\right)
\end{aligned}
$$

and correspond to the initial considered model with $k_{z}=$ $0.1 \mathrm{~m}^{-1} ; \sigma_{v}=0.0345 \mathrm{~m}^{-1}, \beta=\pi / 4 \mathrm{rd}$, and $z_{g}=-1.74 \mathrm{~m}$. The results are also reported for the reduced model described above that leads to $\boldsymbol{T}_{\mathrm{vol}}^{\prime}=\boldsymbol{I}_{d}$ and $\boldsymbol{T}_{\mathrm{gro}}^{\prime}=$ $\operatorname{Diag}[43.8,16.16,11.24]$ and $z_{g}=0$. The same CRB can be observed for the initial and the reduced models. Other examples with different $\boldsymbol{T}_{\mathrm{vol}}, \boldsymbol{T}_{\mathrm{gro}}$, and $z_{g}$ but with identical $\lambda_{1}, \lambda_{2}$, and $\lambda_{3}$ have led to the same perfect superposition and have shown that the value of $z_{g}$ has no influence on the CRB in the range of nonambiguous values. The importance of the eigenvalues of the matrix $\boldsymbol{T}_{\text {vol }}^{-1} \boldsymbol{T}_{\text {gro }}$ (which are equal to $\left(\lambda_{j}\right)_{j=1,2,3}$ ) has been already mentioned in [6], and the present result shows that, with $h_{v}$, they rigorously constitute invariant parameters for the estimation precision characterized by the CRB of $h_{v}$.

\section{APPROXIMATE INVARIANT PARAMETERS}

Assuming that the convention $\lambda_{1} \geq \lambda_{2} \geq \lambda_{3}$ has been adopted, the four parameters of the RVoG model can be chosen as $h_{v}, \mathcal{A}=\left(\lambda_{1}-\lambda_{3}\right) /\left(\lambda_{1}+\lambda_{3}\right), \mathcal{E}=\lambda_{1}+\lambda_{2}+\lambda_{3}$, and $\lambda_{2}$. Parameter $\mathcal{A}$ can be related to the difference of polarimetric behavior of the vegetation and of the ground, whereas $\mathcal{E}$ can be related to the ratio of the energies scattered by the ground and the volume. Indeed, if $\boldsymbol{T}_{\text {gro }}=y \boldsymbol{T}_{\text {vol }}$, where $y$ is a positive real value, then $\mathcal{A}=0$ and $\mathcal{E}=3 y$. Furthermore, if $\boldsymbol{T}_{\mathrm{vol}}=y \boldsymbol{I}_{d}$, then $\mathcal{A}$ is related to the polarimetric response of the ground. 


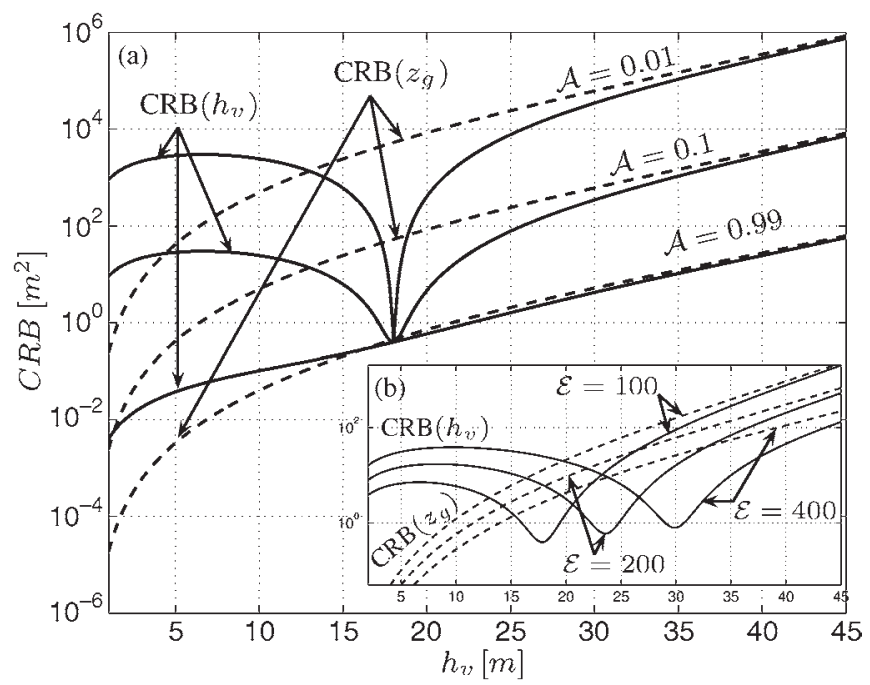

Fig. 2. (a) Same as Fig. 1 but for different values of $\mathcal{A}=\left(\lambda_{1}-\lambda_{3}\right) /\left(\lambda_{1}+\right.$ $\lambda_{3}$ ) when $\mathcal{E}=100$ and for $x=0.2$. (b) Inset: Same as (a) but when $\mathcal{A}=0.2$ and for different values of $\mathcal{E}$.

The influence of $\lambda_{2}$ when $\lambda_{2}(x)=x \lambda_{1}(x)+(1-x) \lambda_{3}(x)$ with $x \in[0,1]$ is illustrated in Fig. 1(b) for the same values of $k_{z}, \beta$, and $\sigma_{v}$ as in Fig. 1(a). For each value of $x, \lambda_{1}(x)$ and $\lambda_{3}(x)$ are determined so that $\mathcal{A}$ and $\mathcal{E}$ remain unchanged. It can be observed, for the considered example, that the influence of $\lambda_{2}$ is low in comparison to the influence of $h_{v}$. An appropriate approximation thus consists in considering that the CRBs of $h_{v}$ and $z_{g}$ are essentially functions of the unknown parameters $h_{v}$, $\mathcal{A}$, and $\mathcal{E}$ and of the known parameters $k_{z}$ and $\alpha=2 \sigma_{v} / \cos \beta$.

Fig. 2 reports the influence of $\mathcal{A}$ and $\mathcal{E}$. The influence of $\mathcal{A}$ is crucial on the CRB values since, for example, when $h_{v}=35 \mathrm{~m}$, the CRB is approximately multiplied by a factor 100 when $\mathcal{A}$ is divided by a factor 10 .

The value $h_{v}$ for which the CRB is minimal has been analyzed in [9] and roughly corresponds to the vegetation height for which the same energy is backscattered by the vegetation and by the ground. It can be seen in Fig. 2(b) that this value of $h_{v}$ is an increasing function of $\mathcal{E}$.

The influence of the height sensitivity $k_{z}$ and of the incident angle $\beta$ is illustrated in Fig. 3. The influence of $k_{z}$ is low, as long as $h_{v}$ is smaller than the ambiguity height $2 \pi / k_{z}$ with the RVoG model that does not take into account instrumental phase noise. Furthermore, the influence of $\beta$ is essentially to modify the value $h_{v}$ for which the CRB $h_{v}$ is minimal.

\section{CONCLUSION}

The group invariance of the homogenous RVoG model shows that four reduced parameters instead of the 20 initial unknown parameters characterize the difficulty of the vegetation and ground height estimation. Moreover, on the considered examples in this letter, only three of them have a nonnegligible influence. The influence of the first parameter $h_{v}$, the vegetation height, was previously analyzed in [9]. This letter shows that the second parameter $\mathcal{A}$ can have a strong influence on the CRB. As illustrated in the proposed examples, high precision can be expected for forest with $\mathcal{A} \simeq 1$, whereas low performance is

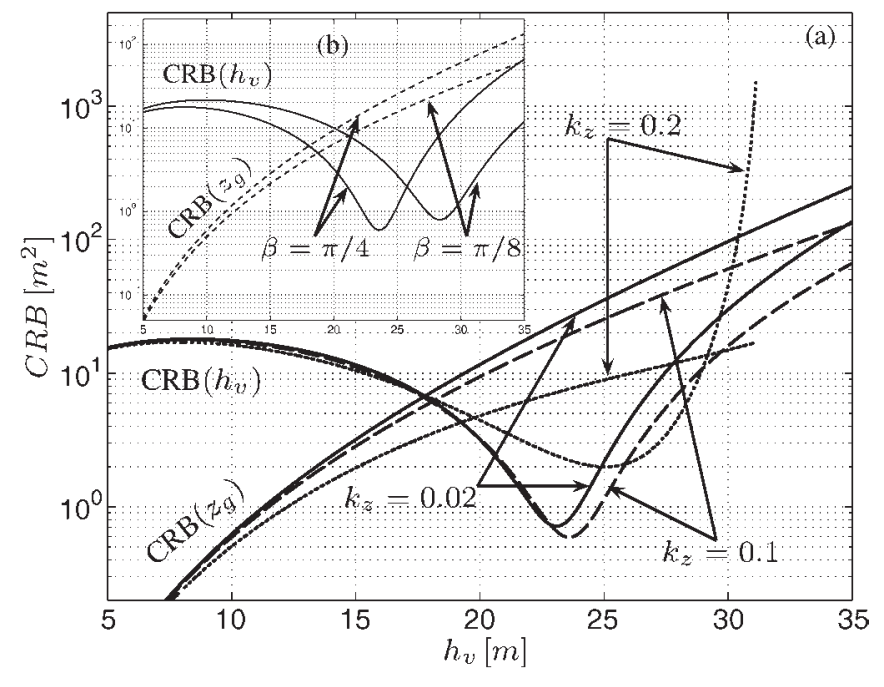

Fig. 3. (a) Same as Fig. $1(x=0.2, \beta=\pi / 4, \mathcal{A}=0.2$, and $\mathcal{E}=200)$ but for different values of $k_{z}$. (b) Inset: Influence of $\beta$.

obtained if $\mathcal{A} \simeq 0$. Indeed, $\mathcal{A}$ can be related to the difference of the polarimetric response between the ground and the volume. The third parameter $\mathcal{E}$ is related to the ratio of the energy scattered by the ground and the volume. The influence of $\mathcal{E}$ is thus important. In particular, it modifies the value of the vegetation height for which the CRB on $h_{v}$ has a local minimum. In the framework of the model, where the instrumental phase noise is not taken into account, the height sensitivity $k_{z}$ seems to have a low influence as long as the vegetation height is smaller than the ambiguity height $2 \pi / k_{z}$, whereas $\beta$ leads to an analogous behavior to the one of $\mathcal{E}$. Any bijection function of the invariant parameters also defines invariant parameters that characterize the difficulty of the estimation task, which can be a useful property to define a mean volume-to-ground ratio that constitutes a rigorous contrast parameter.

\section{APPENDIX}

Since the vectors $\boldsymbol{k}_{j}$ are assumed distributed with a circular zero-mean Gaussian distribution, the vectors $\boldsymbol{k}_{j}^{\prime}=\boldsymbol{M} \boldsymbol{k}_{j}$ are also distributed with a circular zero-mean Gaussian distribution but with a different unknown parameter vector that can be written as $\boldsymbol{\Theta}^{\prime}=g_{M}(\boldsymbol{\Theta})$. Thus, the $\log$ likelihood of observing $\mathcal{K}^{\prime}=$ $\left\{\boldsymbol{k}_{1}^{\prime}, \ldots, \boldsymbol{k}_{N}^{\prime}\right\}$ can be written as $L\left(\mathcal{K}^{\prime} \mid \Theta^{\prime}\right)=L\left(\mathcal{K}^{\prime} \mid g_{M}(\boldsymbol{\Theta})\right)$. However, a direct calculus shows that $L\left(\mathcal{K}^{\prime} \mid \Theta^{\prime}\right)=F(\boldsymbol{M})+$ $L(\mathcal{K} \mid \boldsymbol{\Theta})$, where $F(\boldsymbol{M})$ is a function of $\boldsymbol{M}$ but independent of $\mathcal{K}^{\prime}$ and $\boldsymbol{\Theta}$. Thus, if $\widehat{\boldsymbol{\Theta}}=\left(\widehat{\theta}_{1}, \widehat{\theta}_{2}, \ldots, \widehat{\theta}_{20}\right)$ maximizes $L(\mathcal{K} \mid \boldsymbol{\Theta})$ and if $\widehat{\boldsymbol{\Theta}}^{\prime}=\left(\widehat{\theta}_{1}^{\prime}, \widehat{\theta}_{2}^{\prime}, \ldots, \widehat{\theta}_{20}^{\prime}\right)$ maximizes $L\left(\mathcal{K}^{\prime} \mid \Theta^{\prime}\right)$, then, when there is no estimation ambiguity, $\widehat{\boldsymbol{\Theta}}^{\prime}=g_{M}(\widehat{\boldsymbol{\Theta}})$.

The considered transformation $\boldsymbol{k}_{j}^{\prime}=\boldsymbol{M} \boldsymbol{k}_{j}$ does not modify $h_{v}$ and translates $z_{g}$ with a deterministic value, which implies that $\widehat{\theta}_{1}^{\prime}=\widehat{\theta}_{1}$ and $\widehat{\theta}_{2}^{\prime}=\widehat{\theta}_{2}-z$. The variance of these estimators is thus the same with the samples $\mathcal{K}^{\prime}$ and $\mathcal{K}$, i.e., when the RVoG model corresponds to parameter $\boldsymbol{\Theta}$ or $\boldsymbol{\Theta}^{\prime}=g_{M}(\boldsymbol{\Theta})$.

Finally, since maximum-likelihood estimators are asymptotically efficient (i.e., with a variance that reaches the CRB), the CRBs of $h_{v}$ and $z_{g}$ are also invariant with transformations $\boldsymbol{M}$. 


\section{ACKNOWLEDGMENT}

The authors are grateful to the Conseil Régional ProvenceAlpes-Côte d'Azur (PACA) for taking part in the funding of the Ph.D. of A. Arnaubec.

\section{REFERENCES}

[1] S. R. Cloude, Polarisation: Applications in Remote Sensing. London, U.K.: Oxford Univ. Press, 2009.

[2] J. S. Lee and E. Pottier, Polarimetric Radar Imaging: From Basics to Applications. Boca Raton, FL, USA: CRC Press, 2009.

[3] R. N. Treuhaft, S. N. Madsen, M. Moghaddam, and J. J. van Zyl, "Vegetation characteristics and underlying topography from interferometric radar," Radio Sci., vol. 31, no. 6, pp. 1449-1495, Nov./Dec. 1996.

[4] R. N. Treuhaft and P. R. Siqueira, "Vertical structure of vegetated land surfaces from interferometric and polarimetric radar," Radio Sci., vol. 35, no. 1, pp. 141-177, Jan./Feb. 2000.

[5] K. P. Papathanassiou and S. R. Cloude, "Single-baseline polarimetric SAR interferometry," IEEE Trans. Geosci. Remote Sens., vol. 39, no. 11, pp. 2352-2363, Nov. 2001.

[6] S. R. Cloude and K. P. Papathanassiou, "Three-stage inversion process for polarimetric SAR interferometry," Proc. Inst. Elect. Eng.-Radar Sonar Navig., vol. 150, no. 3, pp. 125-133, Jun. 2003.

[7] F. Garestier, P. Dubois-Fernandez, and I. Champion, "Forest height inversion using high-resolution P-band Pol-InSAR data," IEEE Trans.
Geosci. Remote Sens., vol. 46, pt. 1, no. 11, pp. 3544-3559, Nov. 2008

[8] A. Roueff, A. Arnaubec, P.-C. Dubois-Fernandez, and P. Réfrégier, "Cramer-Rao lower bound analysis of vegetation height estimation with random volume over ground model and polarimetric SAR interferometry," IEEE Geosci. Remote Sens. Lett., vol. 8, no. 6, pp. 1115-1119, Nov. 2011

[9] A. Arnaubec, A. Roueff, P.-C. Dubois-Fernandez, and P. Réfrégier, "Vegetation height estimation precision with compact PolInSAR and homogeneous random volume over ground model," IEEE Trans. Geosci. Remote Sens., vol. 52, no. 3, pp. 1879-1891, Feb. 2014.

[10] P. Garthwaite, I. Jolliffe, and B. Jones, Statistical Inference. London, U.K.: Prentice-Hall, 1995.

[11] T. S. Ferguson, Mathematical Statistics: A Decision Theoretic Approach. New York, NY, USA: Academic, 1967.

[12] C. G. Giri, Group Invariance in Statistical Inference. Singapore: World Scientific, 1996.

[13] P. Réfrégier and F. Goudail, "Invariant polarimetric contrast parameters of coherent light," J. Opt. Soc. Amer. A, Opt. Image Sci., vol. 19, no. 6, pp. 1223-1233, Jun. 2002.

[14] P. Réfrégier, M. Roche, and F. Goudail, "Invariant polarimetric contrast parameters of light with Gaussian fluctuations in three dimensions," J.Opt. Soc. Amer. A, Opt.ImageSci., vol. 23, no. 1, pp. 124-133, Jan. 2006.

[15] J. Morio, P. Réfrégier, F. Goudail, P. Dubois-Fernandez, and X. Dupuis, "Information theory based approach for contrast analysis in polarimetric and/or interferometric SAR images," IEEE Trans. Geosci. Remote Sens., vol. 46, no. 8, pp. 2185-2196, Aug. 2008. 